http://dx.doi.org/10.35381/e.k.v4i8.1355

\title{
Recursos audiovisuales para la enseñanza de lectoescritura
}

\section{Audiovisual resources for teaching literacy}

Tania Valeria Feicán-Zumba

tania.feican.48@est.ucacue.edu.ec

Universidad Católica de Cuenca, Paccha

Ecuador

https://orcid.org/0000-0002-8000-8031

Darwin Gabriel García-Herrera

dggarciah@ucacue.edu.ec

Universidad Católica de Cuenca, Azogues

Ecuador

https://orcid.org/0000-0001-6813-8100

Cristián Andrés Erazo-Álvarez

cristianerazo@ucacue.edu.ec

Universidad Católica de Cuenca, Cuenca

Ecuador

https://orcid.org/0000-0001-8746-4788

Recepción: 15 de marzo 2021

Revisado: 15 de mayo 2021

Aprobación: 15 de junio 2021

Publicación: 01 de julio 2021 


\title{
RESUMEN
}

La pandemia y el aumento del uso de la tecnología ha obligado a los docentes a emplear diferentes estrategias didácticas para mejorar la enseñanza. Por tal motivo los recursos digitales deben ser creativos, motivadores y de calidad que permitan el pensamiento crítico y reflexivo en el aprendizaje. Esta investigación tiene como objetivo determinar cuál es la influencia del uso de recursos audiovisuales para mejorar la lectoescritura en niños de segundo grado de la Unidad Educativa Santa Mariana de Jesús. De tipo descriptiva. La simple utilización de una computadora o celular no es suficiente en la educación si el docente no prepara las herramientas necesarias para crear el interes y captar la atención de los estudiantes. Por ello es imprensindible que los docentes utilicen al menos una herramienta digital en el proceso de aprendizaje.

Descriptores: Motivación; enseñanza asistida por ordenador; aprendizaje en línea. (Palabras tomadas del Tesauro UNESCO).

\begin{abstract}
The pandemic and the increase in the use of technology has forced teachers to use different teaching strategies to improve teaching. For this reason, digital resources must be creative, motivating and of quality that allow critical and reflective thinking in learning. This research aims to determine what is the influence of the use of audiovisual resources to improve literacy in second grade children of the Santa Mariana de Jesús Educational Unit. Descriptive type. The simple use of a computer or cell phone is not enough in education if the teacher does not prepare the necessary tools to create interest and capture the attention of students. For this reason, it is essential that teachers use at least one digital tool in the learning process.
\end{abstract}

Descriptors: Motivation; computer assisted instruction; electronic learning. (Words taken from the UNESCO Thesaurus). 


\section{INTRODUCCIÓN}

La educación incluye a la tecnología como uno de sus recursos didácticos e innovadores en los actuales contextos, así (Torrealba-Peraza, 2004), en las investigaciones realizadas en España se identifica que la multimedia es la integración de medios audiovisuales y que tiene un poder comunicacional directo en las personas, estos recursos didácticos casi siempre se dirigen a una audiencia en específico dependiendo de la materia que se proyecte y que tengan un proceso de mejora continua en el espectador. Realizar recursos audiovisuales hace más productivo la labor educativa, por eso inicia un trabajo colaborativo entre docentes que lo comparten, mejorando la eficacia de contenidos con materiales de calidad.

De otro lado consideran fundamental las tecnologías de la información y comunicación (TIC) en la educación porque no solo se trata de un recurso digital, sino de una estrategia didáctica que se puede aplicar en diferentes destrezas pedagógicas. Las TIC fortalecen la comprensión y creatividad se hace más espontanea debido a que los estudiantes quieren aprender de manera diferente a la tradicional colaborando con la enseñanza que brindan sus docentes. Este enfoque convierte al docente en facilitador o guía, pero es el encargado de seleccionar los contenidos que serán abordados en el proceso de enseñanza aprendizaje.

Dejando así de lado la enseñanza tradicional como indica (Polanco-Garay \& Moré-Soto, 2020), debido que desde marzo del 2020 los docentes tendrían que continuar sus clases de manera virtual, ya sea de forma crónica o asincrónica pero simultáneamente con los estudiantes a fin de seguir con la enseñanza aprendizaje en un espacio y tiempo facilitado por las diferentes tecnologías.

Cabe señalar que en Cuba en la investigación realizada por (Guaña-Moya et al., 2017), se pudo observar un aumento en el uso de la tecnología es así que en el año 2014, se usaba un 54,7\% y para el 2015 el 57\% implicando que el uso del internet y diferentes plataformas se volvieron parte de la vida cotidiana y una herramienta necesaria en la educación, trabajo, negocios. Con el uso del internet las diferentes plataformas digitales 
se han visto obligadas a desarrollar aplicaciones y recursos audiovisuales que mejoren el aprendizaje en la educación para que sean más sencillas en su manejo, además que sean realizadas y aplicadas en tiempo real e interactuar al mismo tiempo con sus pares. En el Ecuador el aumento del uso de redes tecnologías y de comunicación de acuerdo con (Marín-Gutiérrez et al., 2013) estas redes influyen en la vida de las personas directamente hasta llegar al punto en el que la comunicación debe ser rápida, apto de superar barreras demostrando la velocidad y veracidad de las diferentes plataformas. Estos procesos de actualización en las innovaciones tecnológicas han permitido además que las personas pueden acceder al uso de las mismas porque se consigue subscribir de forma gratuita o pagando cantidades considerables debido al crecimiento de forma global en uso de los recursos tecnológicos.

El manejo de las tecnologías aún no se habría mejorado en muchos países, pues habría que decir también que jamás en toda nuestra historia se ha producido un confinamiento total de las instituciones educativas a nivel mundial y en especialmente en Latinoamérica por una cepa que se propago rápidamente. Lo que ha sucedió en Ecuador desde el 2020 con el virus COVID ha provocado que las instituciones y docentes apliquen e incluso se capaciten en el uso y manejo de diferentes plataformas digitales tanto en software y hardware que ayuden a mejorar la calidad de enseñanza en sus estudiantes.

También es cierto lo que (García-Aretio, 2021), dice que los docentes tuvieron inconvenientes al aprender a manejar plataformas virtuales para dar sus clases lo que se transformaría en una educación en línea mediante la utilización de diferentes plataformas como Classroom, zoom, entre otras con el fin de llegar a todos los estudiantes con el proceso de enseñanza a aprendizaje, pero respetando el ritmo y forma de aprendizaje propio de cada estudiante.

El uso y manejo de diferentes plataformas digitales ayudan a determinar cuál es la influencia de estos recursos audiovisuales para mejorar la lectoescritura en niños. Pues las clases online ha creado nuevos retos para los docentes a crear nuevas formas de ayudar en el aprendizaje, es así que han puesto su esfuerzo para introducir nuevas 
estrategias y enseñar en los diferentes niveles de educación; motivando el gusto por la escritura y la lectura con la utilización de diferentes herramientas audiovisuales, en estas se pueden insertan imágenes, palabras y sonidos permitiendo tener una mejor experiencia en el aprendizaje de los estudiantes siendo uno de los mejores recursos didácticos en el siglo XXI.

\section{Referencial teórico}

A partir de la concepción del constructivismo según (Ortiz-Granja, 2015), expresa que hay una interacción entre el docente y los estudiantes, implicando el intercambio de conocimientos entre ambos y de esta manera puede ser productiva, en cierto sentido el resultado obtenido de los contenidos revisados será un aprendizaje significativo. Es decir, en el constructivismo necesariamente debe haber una relación entre metodologías y recursos a utilizar en el proceso de enseñanza aprendizaje para que los estudiantes sean actores principales en la educación.

Pues este estudio se basa en la falta de uso de recursos audiovisuales y la falta de conocimientos de diferentes herramientas por parte de los docentes. El uso de recursos audiovisuales es sumamente importante dado que en las diferentes herramientas digitales se pueden incorporar imágenes, animaciones en movimiento; creando dinamismo en las actividades que se realizan por los docentes y hace más recreativo e interactivo las diferentes aplicaciones creadas para el aprendizaje, mismos que a través de un enlace pueden acceder fácilmente.

Estas aplicaciones audiovisuales brindan la posibilidad de trabajar e incorporar las nuevas tecnologías a las clases de los docentes permitiendo a los estudiantes adquirir aprendizajes de forma digital y formatos virtuales. Es así que el beneficio es dirigido a los estudiantes porque dichos recursos permiten enseñar, fortalecer y retroalimentar contenidos que sean de interés del educando, así (Aguaded-Gómez \& Amor PérezRodríguez, 2012), indican que fomentar el uso de audiovisuales debido a los cambios 


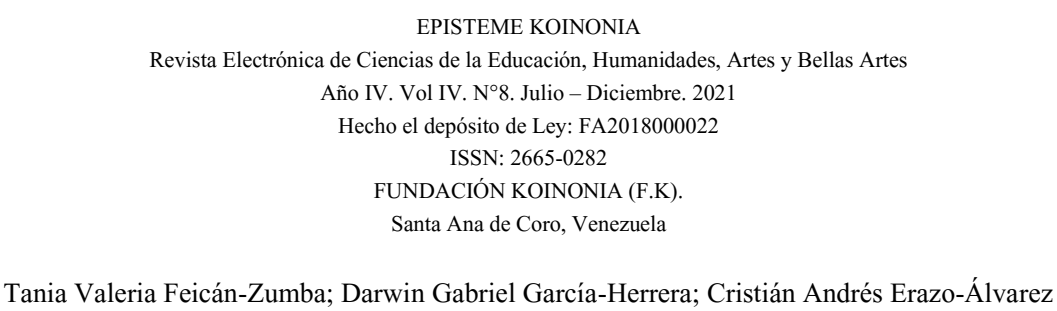

sociales conllevan a un análisis y comprensión de recursos disponibles en las diferentes redes según su necesidad.

Los recursos tecnológicos impulsan a los docentes a formarse o auto educarse para ser innovadores con el uso de las tecnologías; mejorar las estrategias didácticas en los docentes; sintetizar contenidos significativos para el aprendizaje; realizar materiales audiovisuales de acuerdo al interés del educando; captar la atención de los estudiantes; crear recursos de fácil accesibilidad y manejo. Por otro lado, estos recursos son un medio expresivo para transmitir conocimientos, contenidos o mensajes que favorecen a la mejor comprensión y conllevan actitudes críticas y constructivas.

\section{Influencia del uso de recursos audiovisuales para mejorar la lectoescritura}

La influencia de los recursos audiovisuales permite el procesamiento de información de contenidos de una manera más llamativa, eficiente y significativa, aparte de mejorar la lectura y escritura los niños podrán aprender a manejar diferentes plataformas digitales que en un futuro ayudarán a tener mejores experiencias de aprendizaje para crear nuevos contenidos de información para sí mismo y para los que estén en su entorno.

Estos medios audiovisuales y recursos tecnológicos posibilitan una mejor enseñanza y aprendizaje a partir de los lenguajes audio-visuales, mismo que ayudan a mejorar la retención de conocimiento en los estudiantes. También, permite tener a los estudiantes una educación motivadora, participativa, creativa, flexible y dinámica mejorando los estilos de aprendizaje y una mejor interiorización de conocimientos permitiendo tener un aprendizaje propio a través de nuevas experiencias mediante el uso y manejo de recursos audiovisuales. Cabe señalar que los recursos audiovisuales tienen los siguientes elementos:

a) Palabras: que ayudan a entender el contenido y sean ajustadas a la comprensión de los estudiantes.

b) Sonidos: que asocien con lo que se está viendo para mejorar las experiencias y desarrollo su lenguaje de forma lúdica. 
EPISTEME KOINONIA

Revista Electrónica de Ciencias de la Educación, Humanidades, Artes y Bellas Artes

Año IV. Vol IV. Nº. Julio - Diciembre. 2021

Hecho el depósito de Ley: FA2018000022

ISSN: 2665-0282

FUNDACIÓN KOINONIA (F.K)

Santa Ana de Coro, Venezuela

Tania Valeria Feicán-Zumba; Darwin Gabriel García-Herrera; Cristián Andrés Erazo-Álvarez

c) Imágenes: que ayudan a interiorizar un aprendizaje y crear conexiones con lo que se esté aprendiendo.

Al juntar estos recursos crean una interacción entre palabras, sonidos e imágenes en movimiento obteniendo medios audiovisuales que transmiten contenidos determinados de conocimientos que ayudan a mejorar las experiencias de aprendizaje. De estas circunstancias nace el hecho de utilizar recursos audiovisuales en la lectoescritura, en este sentido, (Suárez-Cárdenas et al. 2015), expresan que las narrativas digitales permiten ir estrechamente de la evolución de las tecnologías y la ciencia, ya que involucra el desarrollo de habilidades de lectura y escritura en los niños y transforma la pedagogía tradicional pasiva en un aprendizaje activo y a ser los actores de su aprendizaje.

\section{Aportaciones de los recursos audiovisuales al aprendizaje.}

Las aportaciones de los recursos audiovisuales en el aprendizaje son muy útiles ya que tiene un entorno rico y variado, a partir del cual los estudiantes pueden tener mejores experiencias cuando su docente interactúa y da las correspondientes indicaciones para el correcto uso de ellas. Estos recursos en gran medida deben tener continuidad con las actividades académicas que prepara el docente. Hay que tener en claro que los recursos didácticos permiten involucrarse en cualquier momento de una clase del proceso de enseñanza aprendizaje, más no garantiza una educación autónoma de estudiante por lo que es necesario e indispensable que el docente sea el guía y que permita una reflexión mediante nuevas formas de concebir un aprendizaje con la inserción de tecnologías ajustándose a los cambios de la actualidad y de los estudiantes para que ellos sean beneficiados en la educación.

El empleo de los recursos audiovisuales en el aprendizaje por parte de los estudiantes crea diferentes formas de aprender, así como desarrollar su autonomía e incentiva el gusto por mejorar e incorporar nuevos conocimientos y destrezas de acuerdo a su 
realidad. Teniendo información a la mano en cualquier lugar y tiempo ya que pueden ser acceder gracias al uso del internet.

Los recursos audiovisuales en el transcurso de aprender a leer y escribir han sido un factor de gran ayuda para los niños y niñas que se encuentran en este proceso, pues si bien es cierto deben salir leyendo y escribiendo de los primeros años de escolaridad ellos necesitan de diferentes recursos que motiven el aprendizaje. Estos recursos se han convertido en algo interactivo ya que la mayoría son creados con fines de captar la atención y de crear diversión, pero sin dejar de lado el aprendizaje que se puede conseguir mediante un juego o la observación de videos.

Una herramienta digital que permita el juego a través de la decodificación de palabras, de sopa de letras, de anagramas, permiten que el estudiante juegue, pero a la vez ponga en práctica lo aprendido en el proceso de la lectoescritura porque permite crear habilidades de fluidez guiándose en las ordenes que presenten para iniciar el juego. Los recursos audiovisuales son herramientas que permiten realizar, reproducir y difundir una información o mensajes visuales y sonoros que fortalecen el aprendizaje y los conocimientos, siendo importante realizar adecuadamente y emplear los recursos necesarios para que puedan ser percibidos en un mismo tiempo por los sentidos del oído y la vista del estudiante.

La utilización de recursos audiovisuales hace que los estudiantes sientan atracción por el uso y manejo de dichos recursos que son aplicados en la enseñanza-aprendizaje, por tal motivo es causa de curiosidad en los estudiantes y estimula el pensamiento crítico y reflexivo teniendo agrado y motivación para aprender y sentirse incluidos en el sistema educativo. Es por ello que las instituciones educativas deben crear, desarrollar y utilizar diferentes recursos y estrategias para optimizar la comprensión de contenidos.

Dentro de las ventajas que nos ofrece el uso de recursos audiovisuales son positivos porque despierta el interés, la atención, la participación activa, la comprensión, a ser más críticos evitado el aprendizaje memorístico y pasivo. Pero, además para el docente su 


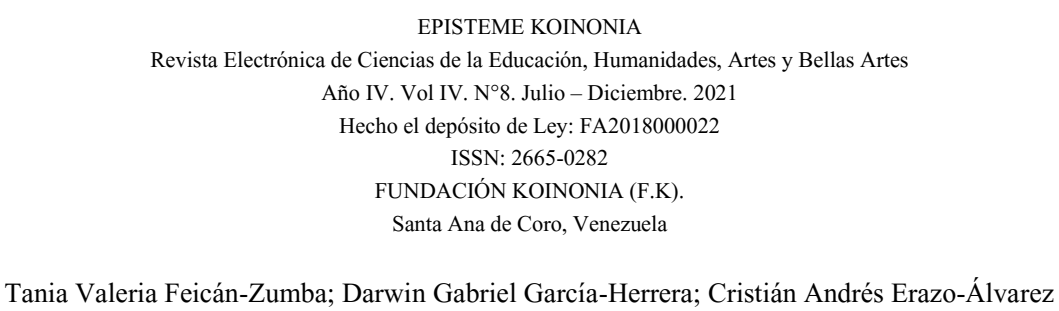

labor se vuelve más llevadera porque es más dinámica, creativa y despierta el interés en sus estudiantes y alejándose de una pedagogía tradicional.

Por otro lado, comprende que las habilidades en lectoescritura en los estudiantes (Ballestas-Camacho, 2015), son fundamentales porque permiten una mejor comprensión de textos escritos de las diferentes asignaturas; tanto la escritura como la lectura permite la transmisión de una o varias ideas o mensajes. Las escuelas y docentes deben implementar las herramientas y recursos digitales necesarios que favorezcan el aprendizaje de lectoescritura en sus estudiantes para que ellos puedan producir y comprender textos ya que los primeros años son indispensables para la formación y el gusto hacia la lectura y de esta manera desarrollarán habilidades autónomas en la lectoescritura.

Es así como (Gómez-Díaz et al. 2015), explican que el aumento de diferentes aparatos tecnológicos y el uso de las mismas ha llevado a reflexionar a los docentes sobre las metodologías y estrategia aplicadas en el proceso de enseñanza de lectoescritura y asegurar el desarrollo de competencias y conocimientos en los niños. Pues es un reto enseñar a escribir y a leer a los niños por tal motivo el docente deber ser creativo al momento de elaborar su material audiovisual para que el contenido sea sencillo, directo y divertido de aprender, ya que la idea es que el estudiante vaya enriqueciendo su vocabulario y ejercitando a la memoria con el aprendizaje de nuevas letras del alfabeto, la fonética, grafías, palabras y textos impulsando la comprensión lectora.

\section{Herramientas que se pueden utilizar en lectoescritura.}

De acuerdo a varias investigaciones sobre estrategias para mejorar la lectura y escritura en niños se comparte que la tecnología es una herramienta que ha permitido mejorar la calidad de aprendizaje de los estudiantes pues muchas de estos recursos son de fácil manejo y sin ningún costo. Es así que entre esas herramientas podemos encontrar a Educandy, YouTube, Genially; estas son herramientas que podemos encontrar en línea, misma que nos permiten diseñar juegos, videos, presentaciones e incluso interactuar al 
mismo tiempo con otras herramientas que se pueden embeber a través de sus links y estas aparezcan en una sola herramienta que se esté presentando tal es el caso de la herramienta de Genially.

Realizar diferentes contenidos en las herramientas audiovisuales es adaptarse a las nuevas tecnologías con las cuales los estudiantes van creciendo, por tanto son nativos digitales que están inmersos en él y el manejo y uso correcto estará en gran medida a responsabilidad de lo que los docentes creen para el transcurso de aprendizaje y así ir interactuando con ellos, además que se van aplicando nuevas metodologías donde el docente actúa como orientador, mediador o guía en el aprendizaje y el actor principal recae directamente en el estudiante.

Recordemos que en la época actual el uso de recursos audiovisuales en el proceso de enseñanza de la lectoescritura tiene influencia en el actuar de los estudiantes. De tal manera que los elementos que se incorporen en las presentaciones audiovisuales tendrán consecuencias directas en fortalecer habilidades y capacidades de los niños. Pues los audiovisuales como recurso se ha vuelto imprescindible porque permite el fortalecimiento de los conocimientos adquiridos en el proceso de enseñanza aprendizaje.

\section{METODOLOGÍA}

Se trabajó metodológicamente con un tipo de investigación descriptiva con diseño no experimental, en una población investigada de 29 padres de familia de la Unidad Educativa Santa Mariana de Jesús para saber cómo ayudó a mejorar la lectoescritura los recursos audiovisuales aplicados en la enseñanza aprendizaje de los niños, aplicándose encuesta online y un cuestionario de 11 preguntas de diversas alternativas de respuestas, siendo validado por análisis de alfa de Cronbach con un 0,711. Los datos recopilados fueron procesados desde la estadística descriptiva. 


\section{RESULTADOS}

La lectoescritura en los niños es una de las etapas más importantes dentro del proceso de su formación académica, pues los conocimientos aprendidos en los primeros años de educación serán indispensables en la escritura y comprensión de diferente información que se encuentren en textos, revistas, cuentos, etc.

Es por esto que se ha realizado un y un análisis de normalidad de datos en la cual se demostró que todas las variables son paramétricas es así que se presentan las siguientes tablas:

Tabla 1.

Aporta los recursos digitales al aprendizaje.

\begin{tabular}{llcccc}
\hline & & & & Porcentaje \\
& & Frecuencia & Porcentaje & Porcentaje válido & acumulado \\
\hline Válidos & Siempre & 12 & 41,4 & 41,4 & 41,4 \\
& Casi siempre & 7 & 24,1 & 24,1 & 65,5 \\
& A veces & 8 & 27,6 & 27,6 & 93,1 \\
& Poco & 2 & 6,9 & 6,9 & 100,0 \\
\cline { 2 - 5 } & Total & $\mathbf{2 9}$ & $\mathbf{1 0 0 , 0}$ & $\mathbf{1 0 0 , 0}$ & \\
\hline
\end{tabular}

Fuente: Cuestionario.

La tabla 1 evidencia que los recursos digitales que en un 93,1\% de los recursos digitales aportan al aprendizaje, por lo que es necesario analizar el uso en conjunto de la tecnología y de esta forma proponer el uso de recursos digitales audiovisuales. 


\section{Tabla 2.}

La lectoescritura con el uso de la tecnología es más...

\begin{tabular}{llcccc}
\hline & & & $\begin{array}{c}\text { Porcentaje } \\
\text { válido }\end{array}$ & $\begin{array}{c}\text { Porcentaje } \\
\text { acumulado }\end{array}$ \\
\hline Válidos & Es más dinámica. & 7 & 24,1 & 24,1 & 24,1 \\
& Es de fácil manejo. & 12 & 41,4 & 41,4 & 65,5 \\
& Lo niños se divierten más. & 1 & 3,4 & 3,4 & 69,0 \\
& Los niños aprenden mejor. & 8 & 27,6 & 27,6 & 96,6 \\
& Es de difícil manejo. & 1 & 3,4 & 3,4 & 100,0 \\
\cline { 2 - 5 } & Total & $\mathbf{2 9}$ & $\mathbf{1 0 0 , 0}$ & $\mathbf{1 0 0 , 0}$ & \\
\hline
\end{tabular}

Fuente: Cuestionario.

La tabla 2 evidencia que el uso de tecnología en un 96,6\% aportan a mejorar la lectoescritura, por lo que es necesario determinar las ventajas que se pueden dar con la utilización de la de las misma y de esta forma proponer herramientas que favorecen un mejor aprendizaje en el proceso de enseñanza.

\section{Tabla 3.}

Los recursos digitales brindan aprendizajes significativos.

\begin{tabular}{llcccc}
\hline & & & Porcentaje & Porcentaje \\
& & Frecuencia & Porcentaje & válido & acumulado \\
\hline Válidos & Muy frecuentemente & 3 & 10,3 & 10,3 & 10,3 \\
& Frecuentemente & 5 & 17,2 & 17,2 & 27,6 \\
& Casi nunca & 9 & 31,0 & 31,0 & 58,6 \\
& Nunca & 12 & 41,4 & 41,4 & 100,0 \\
\cline { 2 - 5 } & Total & $\mathbf{2 9}$ & $\mathbf{1 0 0 , 0}$ & $\mathbf{1 0 0 , 0}$ & \\
\hline
\end{tabular}


Fuente: Cuestionario.

La tabla 3 evidencia que los recursos digitales aportan a un aprendizaje significativo en un $58,6 \%$ en adquirir conocimientos, por lo que es inevitable introducir diferentes herramientas digitales dentro de las metodologías aplicadas por los docentes con el fin de proponer a los estudiantes nuevas maneras de aprendizaje para que se apropien de la información necesaria y optimizar sus conocimientos.

\section{PROPUESTA}

Analizados los cuestionarios recogidos se puede plantear que los recursos audiovisuales se han vuelto indispensables en el proceso de enseñanza-aprendizaje, dado que los estudiantes necesitan de herramientas tecnológicas para poder apoyar las diferentes actividades realizadas por los docentes y por ellos mismos. Estas herramientas a su vez necesitan de tres ejes principales:

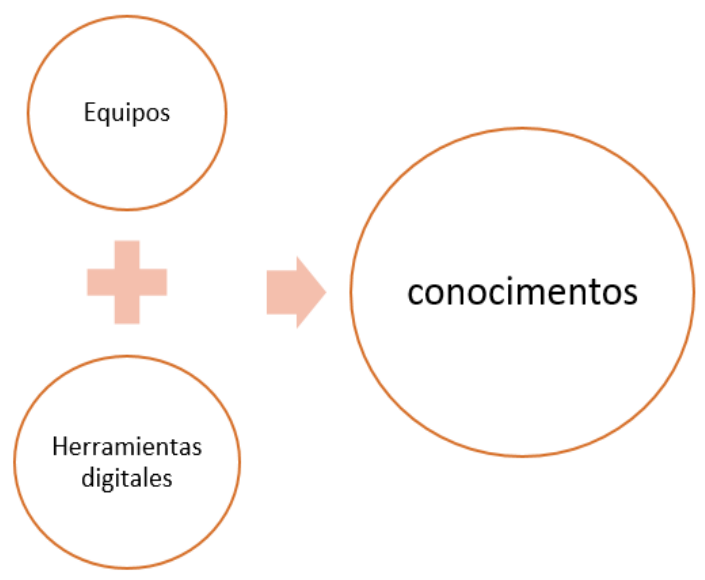

Figura1. Ejes principales de una herramienta digital. Elaboración: Los autores. 
Equipos: que me permita el ingreso a diferentes plataformas desde cualquier dispositivo: computadoras, celulares, iPad, etc., con la facilidad de interactuar en cualquier momento y lugar.

Herramientas digitales: que admitan insertar diferentes tipos de elementos a presentaciones y videos realizados con un fin o propósito para llegar al público. De igual manera que su uso sea manipulable por los diferentes actores de nuestra sociedad, brindando creatividad, imaginación, solución a diferentes problemas que se presentan en la vida estudiantil, además de que ofrezca facilidad y seguridad en lo que se esté creando o desarrollado para futuras visualizaciones y aprendizajes que se puedan dar a través del manejo de cada una de ellas.

Conocimientos: las diferentes tecnologías y herramientas deben brindar los aprendizajes de una información significativa que cause impacto en la adquisición de conocimientos e interiorización en proceso de aprendizaje y de todo lo que se pueda adquirir mediante el uso y manejo de diferentes herramientas tanto visuales como sonoras.

Conviene sin embargo proponer algunas de las herramientas que apoyan y mejoran el aprendizaje de lectoescritura en niños, mismas que en un principio no fueron creadas con esa intencionalidad pero que han ido mejorando según las necesidades que se han presentado en los estudiantes y debido a que estas tienen mucha influencia para mejorar la calidad de aprendizaje, así tenemos:

Educandy: herramienta web gratis, que permite diseñar diferentes juegos educativos visuales para los estudiantes, donde el docente es quien debe registrarse para compartir las actividades recreacionales como: crucigramas, sopa de letras, anagramas, tres en raya, juegos de memoria, ahorcado, etc., y una vez creados los estudiantes podrán 
acceder mediante un link a los diferentes juegos que su docente comparta. Este juego en la lectura ayuda a crear, descubrir y leer palabras reforzando diferentes letras y fonemas para aprender a leer más fluidamente.

Genially: es una herramienta que permite practicar, comprender y disfrutar de una información determinada y a través de la misma permite crear presentaciones, infografías, animaciones, recursos interactivos a través de la gamificación entre otros para los estudiantes de manera virtual.

Los juegos interactivos que ofrece esta herramienta llama la atención y en especial de los niños que están en el proceso de aprender a escribir y leer porque se puede crear, juegos con letras, sílabas, sílabas compuestas que permiten afianzar y profundizar la lectura y escritura. Además, que la lectura no termina solo con la decodificación de palabras sino también con la lectura comprensiva que permite practicar lo aprendido en el proceso de aprendizaje y Genially lo permite hacer mediante la gamificación.

\section{CONCLUSIONES}

Es evidente que la simple utilización de una computadora o celular no es suficiente en la educación si el docente no prepara las herramientas necesarias para crear el interes y captar la atención de los estudiantes. Por ello es imprensindible que los docentes utilicen al menos una herramienta digital en el proceso de aprendizaje, por cuanto hace que los conocimientos que se brinden a los estudiantes sean más relevante e interesante desarronllando diferentes competencias en ellos. La herramientas digitales son fuentes y recursos utiles en la cual los estudiantes pueden utilizar para crear nuevos contenidos 0 para retroalimentar conocimientos adquiridos con anterioridad.

Hay que mencionar además que la población investiga considera que los recursos digitales aportan e influyen en el aprendizaje de los niños y que además estas herramientas las hacen más dinámicas ayudando a mejorar la lectoescritura de los niños porque al reconocer letras y fonemas se les hacen más fácil escribir de manera correcta. 


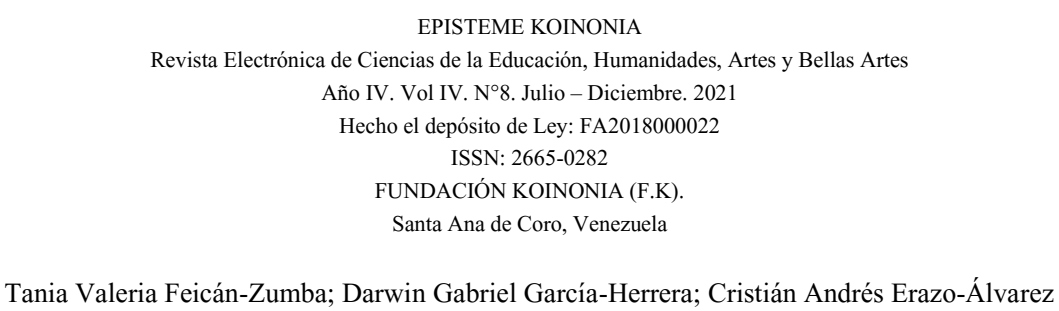

Se considera que los aportes de los recursos digitales son significativos ya que, en la realidad el uso de dichas herramientas cada vez son más las experiencias de los padres de familia y los estudiantes los que avalan que el aprendizaje se vuelve más llevadero y dinámicos porque muestra beneficios con el uso de las tecnologías.

Como resultados se ha propuesto incluir dentro de las metodologías el uso de recursos digitales audiovisuales que ayuden a mejorar el proceso de enseñanza aprendizaje dejando de lado la educación tradicional para que los estudiantes sean los actores principales dentro de la educación y los docentes sean guías o mentores en la utilización de algunas herramientas como Educandy y Genially.

\section{FINANCIAMIENTO}

No monetario.

\section{AGRADECIMIENTO}

A la Corporación Eléctrica del Ecuador y la Jefatura de Posgrados de la Universidad Católica de Cuenca por permitir el desarrollo y fomento de la investigación.

\section{REFERENCIAS CONSULTADAS}

Aguaded-Gómez, I., \& Pérez-Rodríguez, M. (2012). Strategies for media literacy: Audiovisual skills and the citizenship in Andalusia. Journal of New Approaches in Educational Research, 1(1), 22-26. http://dx.doi.org/10.7821/naer.1.1.22-26

Ballestas-Camacho, R. (2015). Relación entre tic y la adquisición de habilidades de lectoescritura en alumnos de primer grado de básica primaria [Relationship between tic and the acquisition of literacy skills in first grade elementary school students]. Investigación 368. https://doi.org/10.14482/indes.23.2.7398

García-Aretio, L. (2021). COVID-19 y educación a distancia digital: preconfinamiento, confinamiento y posconfinamiento [COVID-19 and digital distance education: preconfinement, confinement and post-confinement]. RIED. Revista Iberoamericana De Educación a Distancia, 24(1), 09-32. https://doi.org/10.5944/ried.24.1.28080 
Gómez-Díaz, R., García-Rodríguez, A., \& Cordón-García, J. A. (2015). Aprender a leer y escribir: aplicaciones para el aprendizaje de la lectoescritura [Learning to read and write: applications for learning to read and write]. Education in the Knowledge Society (EKS), 16(4), 118-137. https://doi.org/10.14201/eks2015164118137

Guaña-Moya, C., Quinatoa-Arequipa, J., Pérez-Fabara, E., \& Augusta, M. (2017). Tendencias del uso de las tecnologías y conducta del consumidor tecnológico [Trends in the use of technologies and behavior of technological consumers]. Ciencias Holguín, 23(2), 1-17. https://n9.cl/yeorh

Marín-Gutiérrez, I., Díaz-Pareja, E., \& Aguaded, I. (2013). La competencia mediática en niños y jóvenes: La visión de España y Ecuador [Media competence in children and young people: the vision of Spain and Ecuador]. Chasqui. Revista Latinoamericana de Comunicación, (124),41-47

Ortiz-Granja, D. (2015). El constructivismo como teoría y método de enseñanza. [Constructivism as theory and teaching method]. Sophia, 19(2), 93-110. https://doi.org/10.17163/soph.n19.2015.04

Pereira-Pérez, Z. (2011). Los diseños de método mixto en la investigación en educación: Una experiencia concreta [Mixed method designs in educational research: $A$ concrete experience]. Revista Electrónica Educare, XV(1),15-29.

Polanco-Garay, L.W, Moré-Soto, D. (2021). From traditional learning to Flipped learning as a continuity of the educational process in the context of COVID-19 [Del aprendizaje tradicional al aprendizaje invertido como continuidad del proceso educativo en contexto de COVID-19]. https://n9.cl/5cqx0

Suárez-Cárdenas, I., Celestino Mutis, J., Claudia Yaneth, A., et al. (2015). Desarrollo de la lectoescritura mediante TIC y recursos educativos abiertos [Development of the lecto-writing by means of TICs and educational opened resources]. Apertura, $7(1), 1-13$.

Torrealba-Peraza, J. C. (2004). Aplicación eficaz de la imagen en los entornos educativos basados en la web [Effective application of imaging in web-based educational environments]. Universitat Politècnica de Catalunya. Retrieved from http://www.tdx.cat/handle/10803/6828 
EPISTEME KOINONIA

Revista Electrónica de Ciencias de la Educación, Humanidades, Artes y Bellas Artes

Año IV. Vol IV. No8. Julio - Diciembre. 2021

Hecho el depósito de Ley: FA2018000022

ISSN: $2665-0282$

FUNDACIÓN KOINONIA (F.K)

Santa Ana de Coro, Venezuela

Tania Valeria Feicán-Zumba; Darwin Gabriel García-Herrera; Cristián Andrés Erazo-Álvarez

(02021 por los autores. Este artículo es de acceso abierto y distribuido según los términos y condiciones de la licencia Creative Commons Atribución-NoComercial-Compartirlgual 4.0 Internacional (CC BY-NC-SA 4.0)

(https://creativecommons.org/licenses/by-nc-sa/4.0/). 